\title{
Inducible cAMP Early Repressor Regulates Corticosterone Suppression after Tricyclic Antidepressant Treatment
}

\author{
Alana C. Conti, ${ }^{1}$ Yuo-Chen Kuo, ${ }^{1}$ Rita J. Valentino, ${ }^{3}$ and Julie A. Blendy $y^{1,2}$ \\ ${ }^{1}$ Department of Pharmacology and ${ }^{2}$ Center for Neurobiology and Behavior, University of Pennsylvania, and ${ }^{3}$ The Children's Hospital of Philadelphia, \\ Abramson Pediatric Research Center, Philadelphia, Pennsylvania 19104
}

The cAMP-response element binding protein (CREB) is involved in antidepressant action, but the role of related CRE-binding transcription factors in the behavioral and endocrine responses to antidepressants is unclear. Alternative transcription of the cAMP response element-modulator (CREM) gene yields activator and repressor isoforms, including the strong repressor inducible cAMP early repressor (ICER). ICER is highly expressed in hypothalamic tissues and upregulated after electroconvulsive seizure. Thus, ICER may be a novel mediator of antidepressant action at endocrine and/or behavioral levels. Here we establish that both subchronic and chronic desipramine (DMI) treatments upregulate hypothalamic ICER expression in wild-type mice. Behavioral responses to DMI in the forced swim and tail suspension tests are unchanged in mice lacking ICER. However, the ability of DMI to suppress an acute corticosterone response after swim stress is compromised in ICER-deficient mice, suggesting that increased hypothalamic ICER mRNA after DMI treatment may be required for suppression of corticosterone release. To investigate the mechanism underlying this response, we measured corticotropin releasing factor (CRF), an upstream modulator of corticosterone release. Using real-time quantitative PCR, we establish that hypothalamic CRF expression is significantly reduced after swim exposure in DMI-treated wild-type mice, however DMI is unable to blunt hypothalamic CRF expression in ICER-deficient mice. Furthermore, we demonstrate that ICER is enriched in CRF-expressing neurons in the paraventricular nucleus of the hypothalamus. These data indicate that ICER is required for DMI to reduce stress-induced corticosterone release through regulation of hypothalamic CRF expression, revealing a novel role for ICER in antidepressant regulation of the hypothalamic-pituitary adrenal axis.

Key words: ICER; CREM; corticotropin releasing factor; stress; antidepressant; desipramine; corticosterone; real-time quantitative PCR; $\mathrm{CRH}$; forced swim test; tail suspension test

\section{Introduction}

Antidepressant drugs are widely prescribed and often effective in treating clinical depression; however, the mechanisms of action that underlie long-term therapeutic benefits remain poorly understood. The focus of research has shifted from acute drug action on neurotransmitter transporters to long-term cellular changes that regulate gene expression. Long-term adaptations, including alterations in intracellular signal transduction cascades, are believed to be critical for the efficacy of chronic antidepressant treatment. Identification of such intracellular signaling cascades and downstream gene targets is necessary to understand the complexities associated with clinical depression and will facilitate the development of more effective therapies.

Alterations in the cAMP signaling pathway have been implicated in the mechanism of chronic antidepressant treatment (Nibuya et al., 1996; Dowlatshahi et al., 1998, 1999; Thome et al.,

\footnotetext{
Received 0ct. 24, 2003; revised Dec. 27, 2003; accepted Dec. 29, 2003.

This work was supported by a National Alliance for Research on Schizophrenia and Depression Young Investigator grant, National Institute on Drug Abuse Grant DA-1169-01A2 (J.A.B.), and National Institute of Mental Health Grant MH02006 (R.J.V.). We thank Misty Godfrey and Lindsey Ciali for their technical expertise.

Correspondence should be addressed to Julie A. Blendy, Department of Pharmacology, 125 John Morgan, 3620 Hamilton Walk, Philadelphia, PA 19104-6084. E-mail: blendy@pharm.med.upenn.edu.

DOI:10.1523/JNEUROSCI.4804-03.2004

Copyright $\odot 2004$ Society for Neuroscience $\quad$ 0270-6474/04/241967-09\$15.00/0
}

2000; Conti et al., 2002). The cAMP signaling pathway exerts its transcriptional effects through a family of transcription factors (TFs) that bind to cAMP- responsive elements (CREs), including CRE-binding protein (CREB), CRE modulator (CREM), and Activating TF-1 (ATF-1) (Gonzalez and Montminy, 1989; Lee and Masson, 1993; Molina et al., 1993; Lalli and Sassone-Corsi, 1994). CREB remains the most widely studied member of the CREbinding transcription factor family and has been implicated in the mechanisms underlying antidepressant drug efficacy (Nibuya et al., 1996; Dowlatshahi et al., 1998; Conti et al., 2002). However, the role of related CRE-binding transcription factors in antidepressant action has not been evaluated.

The CREM gene is multiexonic and produces several tissuespecific protein variants known to activate as well as inhibit cAMP-responsive gene expression. The primary CREM activator isoform, CREM $\tau$, is highly expressed in the testes with little expression in brain (Foulkes et al., 1992). Transcription at an alternative promoter site yields a powerful tissue-specific repressor isoform of CREM, the inducible cAMP early repressor (ICER). Unlike CREB and CREM $\tau$, ICER does not contain a kinase-inducible domain and therefore is regulated by changes in its expression level, not by phosphorylation. ICER is classified as a unique early response transcription factor because of its dynamic, and direct cAMP inducibility, which is believed to be 
regulated via four tandem CRE sequences in the ICER promoter (Molina et al., 1993). ICER is the most highly expressed CREM isoform in neuroendocrine tissues (Mellstrom et al., 1993; Molina et al., 1993; Stehle et al., 1993), with cell-specific expression in magnocellular neurons of the hypothalamus (Mellstrom et al., 1993; Luckman and Cox, 1995). Furthermore, ICER expression is significantly upregulated in the pituitary and adrenal glands after hypothalamic-pituitary-adrenal (HPA) axis stimulation (Della Fazia et al., 1998; Mazzucchelli and Sassone-Corsi, 1999). In addition to endocrine regulation of ICER, adrenergic stimulation and electroconvulsive seizures increase ICER expression in brain regions associated with antidepressant efficacy, suggesting a role for ICER in the actions of antidepressant therapies (Fitzgerald et al., 1996a,b).

In this study, we examined the effect of subchronic and chronic DMI administration on ICER mRNA in the hypothalamus of wild-type mice. Using ICER-deficient mice, we investigated whether the observed upregulation of ICER is of functional consequence for either the behavioral or endocrine response to chronic antidepressant treatment. We show that the behavioral responses to antidepressants, as well as stress-induced elevations in corticosterone levels, are independent of ICER expression. However, the ability of antidepressant drugs to block corticosterone release in response to stress is severely impaired in the absence of ICER. Our study demonstrates that ICER is enriched in cells of the paraventricular nucleus and is essential for the ability of antidepressants to regulate the stress-induced activation of the hypothalamic-pituitary-adrenal axis. Together, these studies establish for the first time a role for antidepressant-induced ICER in the regulation of this neuroendocrine system.

\section{Materials and Methods}

Animals. The derivation of mice lacking the CREM gene, including the ICER isoform, has been described previously (Blendy et al., 1996). All isoforms of the CREM gene are deleted in CREM mutant mice. This null allele is maintained in two separate inbred mouse strains, $129 \mathrm{SvEv}$ and C57BL/6, and has been backcrossed for several generations (N11 and N14, respectively) to establish congenic strains. For all experiments described here, CREM mutants and wild-type controls are F1 hybrids obtained from crossing mice heterozygous for the null allele from each parental inbred strain (129SvEv and C57BL/6). Thus, in the F1 generation, all mice are heterozygous for all loci that differ between the parental strains and are genetically identical with the exception of the CREM locus. This breeding scheme allows for rigorous control of the genetic background of experimental animals over time and is in agreement with the recommendations of the Branbury Conference on Genetic Background in Mice (Silva et al., 1997). All mice (3-5 months old, 23-40 gm) were group-housed and maintained on a $12 \mathrm{hr}$ light/dark cycle with food and water available ad libitum in accordance with the University of Pennsylvania Animal Care and Use Committee. All experimental testing sessions were conducted between 12:00 P.M. and 6:00 P.M., with animals randomly assigned to treatment conditions and tested in counterbalanced order.

Drugs. Desipramine (DMI; Sigma, St. Louis, MO) was freshly prepared before use and injected intraperitoneally using a volume of $10 \mathrm{ml} / \mathrm{kg}$. All drug doses were calculated as milligram per kilogram base weight and were dissolved in $0.9 \%$ saline.

Forced swim test. To facilitate adaptation to novel surroundings, mice were transported to the testing room at least $1 \mathrm{hr}$ before testing. Briefly, swim sessions were conducted by placing mice in individual glass cylinders $(46 \mathrm{~cm}$ tall $\times 20.5 \mathrm{~cm}$ diameter $)$ containing $23-25^{\circ} \mathrm{C}$ water at a depth of $10 \mathrm{~cm}$. Whereas the procedure was similar to that described by Porsolt et al. (1977) for rats, a cylinder of larger diameter was used because a larger tank diameter increases predictive validity in the mouse forced swim test (FST) (Sunal et al., 1994). Subjects were given a 15 min preswim, occurring $23.5 \mathrm{hr}$ before the first test swim. On test days a $6 \mathrm{~min}$ test duration was used, and the water was changed between subjects. All test sessions were recorded by a video camera positioned directly above the cylinders. Videotapes were scored by a trained observer who was blinded to the experimental conditions. The behavioral measure scored was the duration of "immobility", defined as the state in which mice were judged to be making only the movements necessary to keep their head above water.

Saline or DMI was administered subchronically by giving three injections over a $24 \mathrm{hr}$ period ( $n=6-12$ per group). Doses were given at 23.5, 5.0 , and $1.0 \mathrm{hr}(10,10$, and $20 \mathrm{mg} / \mathrm{kg}$, s.c., respectively) before the first test swim. Data obtained from pilot experiments showed robust behavioral changes after this dosing strategy. For chronic experiments animals were maintained on saline or DMI injections twice daily $(10 \mathrm{mg} / \mathrm{kg}$, i.p.) for $21 \mathrm{~d}$. On day 21, morning injections were administered $5 \mathrm{hr}$ before FST, and afternoon injections (saline or $20 \mathrm{mg} / \mathrm{kg}$ DMI, s.c.) were administered $1 \mathrm{hr}$ before FST. This dosing regimen was used to allow for comparison of behavioral data obtained from subchronic and chronic studies.

Tail suspension test. Animals were tested in a modified version of the tail suspension test (TST) that has been previously validated (Steru et al., 1985). All mice were adapted to novel surroundings and tested as described for the FST. Subjects were each given saline, DMI, or fluoxetine (FLX; $20 \mathrm{mg} / \mathrm{kg}$, i.p.; $n=8-10$ per group). Thirty minutes after injection, mice were individually suspended by the tail to a horizontal ring-stand bar (distance from floor $=35 \mathrm{~cm}$ ) using Fisherbrand (Fisher Scientific, Pittsburgh, PA) adhesive tape affixed $2 \mathrm{~cm}$ from the tip of the tail. The dosing paradigm was selected from previous studies showing robust behavioral changes under these conditions (Lucki, 2001; Lucki et al., 2001). Mice demonstrated several escape-oriented behaviors interspersed with bouts of immobility as the session progressed. A 6 min test session was used that was recorded by a video camera positioned in front of the tail suspension apparatus. Videotapes were scored by a trained observer who was blinded to the experimental conditions. The behavioral measure scored was the duration of "immobility", defined as the time when mice were judged to cease escape-motivated behaviors.

RNA isolation and cDNA synthesis. After cervical dislocation, brains were dissected for RNA analysis ( $n=6-12$ per group). All RNA was isolated using TRIzol (Invitrogen, Gaithersburg, MD) according to manufacturer's instructions. The quality of the RNA samples was determined by ethidium bromide staining of $18 \mathrm{~S}$ and $28 \mathrm{~S}$ rRNAs after fractionation on denaturing agarose gels. Contaminating genomic DNA was removed using $1 \mu \mathrm{l}$ of RNase-free DNase I (Boehringer Mannheim, Indianapolis, IN) $/ 10 \mu \mathrm{g}$ of RNA at $37^{\circ} \mathrm{C}$ for 30 min. cDNA was synthesized using Moloney murine leukemia virus-reverse transcriptase (Invitrogen) with deoxyribonucleotides (dNTPs) and random hexamer primers RT (Invitrogen) at $42^{\circ} \mathrm{C}$ for $30 \mathrm{~min}$.

A subset of animals was used to measure the time course of ICER expression after DMI treatment. Animals were killed at $1 \mathrm{hr}$ after the last DMI or saline injection ( $n=6-7$ per group) to determine effects of subchronic DMI treatment. Animals were killed following $21 \mathrm{~d}$ of saline or DMI administration to determine effects of chronic treatment. To measure corticotropin-releasing factor (CRF) expression after swim exposure, animals were killed $1 \mathrm{hr}$ after FST testing on day 21 of chronic treatment.

RT-PCR. Conditions used for RT-PCR followed the method of Wilson and Melton (1994) with minimal modifications. The mouse hypoxanthine guanine phosphoribosyl transferase (HPRT) gene was used as an internal control, because the mRNA of this gene is expressed in most tissues in approximately equal amounts. cDNAs synthesized from hypothalamic, hippocampal, and cortical RNA provided templates for PCRs under the following conditions: one cycle of $95^{\circ} \mathrm{C}$ for $5 \mathrm{~min}$, followed by 25 cycles of $95^{\circ} \mathrm{C}$ for $1 \mathrm{~min}, 60^{\circ} \mathrm{C}$ for $1 \mathrm{~min}, 72^{\circ} \mathrm{C}$ for $2 \mathrm{~min}$, and one cycle of $72^{\circ} \mathrm{C}$ for $5 \mathrm{~min}$ in a buffer containing $1.5 \mathrm{mmol} / \mathrm{l} \mathrm{MgCl}_{2}, 10 \mu \mathrm{mol} / \mathrm{l}$ primers, $0.05 \mu \mathrm{Ci}{ }^{32} \mathrm{P}$-dATP, and $200 \mu \mathrm{mol} / 1 \mathrm{dNTPs}$. The forward and reverse primers used were as follows: Icer-1 $\gamma(222 \mathrm{bp})$ and Icer -1 (258 bp): 5' -CCCAACATGGCTGTAACTGGA (forward) and 5'-GCACAGCCACACGATTTTCAA (reverse); Hprt (111 bp): 5' -GGCCATCTGCCTAGTAAAGCT (forward) and 5'-GCTGGCCTATAGGCTCATAGT(reverse). 
PCR products were separated on 5\% acrylamide gels that were dried and exposed to PhosphorImager screens overnight. Combined signal intensity of ICER amplification products (ICER-1 + ICER-1 $\gamma$ ) was normalized to that of HPRT. Under the conditions used, determination of mRNA levels by RT-PCR was quantitative, as PhosphorImager analysis (ImageQuant; Molecular Dynamics, Sunnyvale, CA) of amplified products from serially diluted cDNAs confirmed the linearity of the assay for all primers used (data not shown).

Real-time quantitative PCR. Primers and fluorescent probes were designed using the Primer Express 1.5 software (Applied Biosystems, Foster City, CA) and purchased from IDT, Inc. (Coralville, IA) or Invitrogen. The mouse TATA box binding protein (TBP) (Tamura et al., 1991) or cyclophilin A genes were used as internal controls, because the mRNAs of these genes are expressed in most tissues in approximately equal amounts. Sequences for primers and probes are as follows: TBP primers: 5'-CCCCTTGTACCCTTCACCAAT (forward) and 5' - GAAGCTGCGGTACAATTCCAG (reverse); TBP fluorogenic probe: 5' -TCCTGCCACACCAGCTTCTGAGA; Cyclophilin A primers: 5'-GCATACAGGTCCTGGCATCTT (forward) and 5' -CATTCAGTCTTGGCAGTGCAG (reverse); Cyclophilin A fluorogenic probe: 5'-TGGAGCGTTTTGGGTCCAGGA;ICERprimers:5' -GGTGACATGCCAACTTACCAGA(forward) and 5' -TTGCGACTTGCTTCTTCTGC (reverse); ICER fluorogenic probe: $5^{\prime}$-TGATGGCTGCCTCACCAGGAA; CRF primers: 5' -CCGGGCAGAGCAGTTAGC (forward) and 5' -CAACATTTCATTTCCCGATAATCTC (reverse); CRF fluorogenic probe: 5' -CAGCAAGCTCACAGCAACAGGAAACTGAT.

The TBP and cyclophilin A probes were labeled at the $5^{\prime}$ end with the reporter fluorophore, $\mathrm{Cy} 3$, the ICER probe was labeled at the $5^{\prime}$ end with the reporter fluorophore Cy5, and the CRF probe was labeled at the 5' end with the reporter fluorophore, FAM. All probes were labeled at the $3^{\prime}$ end with dark quenchers (BH-2 for ICER, TBP and cyclophilin A, and $\mathrm{BH}-1$ for CRF). To prevent probe extension, phosphate groups were attached to the $3^{\prime}$ ends of all probes. Multiplex PCR conditions were optimized and used as follows: one cycle of $95^{\circ} \mathrm{C}$ for $10 \mathrm{~min}$, followed by 40 cycles of $95^{\circ} \mathrm{C}$ for $30 \mathrm{sec}$ and $60^{\circ} \mathrm{C}$ for $1 \mathrm{~min}$. Amplicons were synthesized and quantified using a Stratagene (La Jolla, CA) MX4000 thermocycler system. Each sample was analyzed in triplicate using $1 \times$ TaqMan Universal PCR Master Mix (Applied Biosystems) using $300 \mathrm{~nm}$ of each TBP primer, $100 \mathrm{~nm}$ fluorogenic TBP probe, $300 \mathrm{~nm}$ CRF forward primer, $900 \mathrm{~nm}$ CRF reverse primer, and $250 \mathrm{~nm}$ fluorogenic CRF probe or using $300 \mathrm{~nm}$ of each cyclophilin A primer, $250 \mathrm{~nm}$ fluorogenic cyclophilin A probe, 300 nм ICER forward primer, 900 nм ICER reverse primer, and $250 \mathrm{~nm}$ fluorogenic ICER probe in each replicate. Data were analyzed using the comparative Ct method, as described in Applied Biosystems User Bulletin Number 2 (P/N 4303859) and detailed by Livak and Schmittgen (2001). For each replicate, an amplification plot was generated displaying the increase in the reporter dye fluorescence with each cycle of PCR. From these data, Ct, defined as the fractional cycle number at which the fluorescence passes a fixed threshold (based on the baseline data collected in the first 15 cycles), was calculated. Therefore, for example, for each sample an average $\mathrm{Ct}$ for $\mathrm{CRF}\left(\mathrm{Ct}_{\mathrm{CRF}}\right)$ and $\mathrm{TBP}\left(\mathrm{Ct}_{\mathrm{TBP}}\right)$ was obtained. Samples were normalized against TBP using the following equation:

$$
C t_{\mathrm{TBP}-\mathrm{CRF}}=\operatorname{mean}\left(\mathrm{Ct}_{\mathrm{TBP}}\right)-\operatorname{mean}\left(\mathrm{Ct}_{\mathrm{CRF}}\right) .
$$

$\Delta \mathrm{Ct}_{\mathrm{TBP}-\mathrm{CRF}}$ was calculated for each experimental group and differences between groups were calculated using the following equation as an example:

$$
C t_{\mathrm{CRF}}=\text { wild-typesaline }\left(\mathrm{Ct}_{\mathrm{TBP}-\mathrm{CRF}}\right) \text { - wild-typeDMI }\left(\mathrm{Ct}_{\mathrm{TBP}-\mathrm{CRF}}\right) \text {, }
$$

where $\Delta \Delta \mathrm{Ct}_{\mathrm{CRF}}$ represents the difference in CRF expression between experimental groups compared. The magnitude of difference in CRF gene expression is equal to $2 \Delta \Delta \mathrm{CtCRF}$. Relative CRF expression levels were normalized to TBP and plotted relative to expression in salinetreated wild-type mice, which equals 1 . ICER expression was normalized to cyclophilin A expression and plotted in the same manner.

Plasma corticosterone assays. On days 1 and 21, tail blood was collected from each animal 20 min after FST test session ( $n=6-12$ per group), separated into plasma by centrifugation and stored at $-20^{\circ} \mathrm{C}$ until assayed. Plasma corticosterone was measured by radioimmunoassay using a commercially available kit (ICN Biomedicals Inc., Cleveland, $\mathrm{OH}$ ). Intra-assay coefficient of variation was $<20 \%$ (average coefficient of variation of $4.71 \%$ ).

CRF immunohistochemistry. Mice were injected intracranially with 2 $\mu \mathrm{l}$ of colchicine $(20 \mathrm{mg} / \mathrm{ml})$ and transcardially perfused $48 \mathrm{hr}$ later with $25 \mathrm{ml}$ of $0.1 \mathrm{M}$ PBS followed by $60 \mathrm{ml}$ of cold $4 \%$ paraformaldehyde. The brains were removed and postfixed for $24 \mathrm{hr}$ at $4^{\circ} \mathrm{C}$, then placed in $30 \%$ sucrose in $0.1 \mathrm{M}$ PBS for at least $48 \mathrm{hr}$. Frozen-thin sections $(10 \mu \mathrm{m})$ were cut on a cryostat and mounted on glass slides (Fisher Plus Probe-on; Fisher Scientific). Sections were processed to visualize CRF immunoreactivity as previously described (Valentino et al., 1992). Briefly, sections were incubated in rabbit anti-CRF antiserum (PBL rC70, preabsorbed with $2 \mathrm{mg}$ human a globulins and $1 \mathrm{mg}$ a-MSH per $\mathrm{ml}$ serum, provided by Dr. Wylie Vale, The Salk Institute, San Diego, CA) at a 1:8000 dilution in $0.1 \mathrm{M}$ PBS containing $0.3 \%$ Triton $\mathrm{X}-100$ and $0.04 \%$ bovine serum albumin (PBST-BSA) and $0.1 \%$ sodium azide for $48 \mathrm{hr}$ at $4^{\circ} \mathrm{C}$. The specificity of this antisera has been previously described (Valentino et al., 1992; Van Bockstaele et al., 1996). The primary antibody was applied directly to the slide containing the sections, and another slide was used to cover the reaction. Sections were rinsed in PBST-BSA $(3 \times 10 \mathrm{~min})$ and incubated in biotinylated donkey anti-rabbit antiserum (1:200 in PBSTBSA) for $90 \mathrm{~min}$ at room temperature. The sections were then rinsed in PBS and incubated in avidin-biotin complex (ABC Elite kit; Vector Laboratories, Burlingame, CA). They were then rinsed in PBS $(3 \times 10 \mathrm{~min})$ and immersed in $0.02 \% 3,3^{\prime}$-diaminobenzidine- $4 \mathrm{HCl}$ (DAB; Sigma) containing $0.01 \% \mathrm{H}_{2} \mathrm{O}_{2}$ in $\mathrm{PB}$ for $10-15 \mathrm{~min}$ at room temperature. All solutions were made using diethylpyrocarbonate-treated reagents.

Paraventricular nucleus microdissection and aRNA amplification. After CRF immunohistochemistry, stained tissue sections were photographed and then treated with $200 \mu \mathrm{l}$ of $500 \mu \mathrm{g} / \mathrm{ml}$ proteinase $\mathrm{K}$ for $30 \mathrm{~min}$ at $42^{\circ} \mathrm{C}$. The localized region of CRF-expressing neurons of the paraventricular nucleus (PVN) was microdissected under bright-field visualization with a Zeiss (Frankfurt, Germany) Axiovert 25 microscope using an Eppendorf MicroDissector equipped with an Eppendorf MicroChisel that was positioned using a joystick micromanipulator. The microdissected tissue was aspirated by pipette and transferred to a microfuge tube. Sections were photographed after microdissection. Microdissected tissue from four wild-type mice was concentrated to a volume of $10 \mu \mathrm{l}$. RNA amplification was carried out using the MessageAmp aRNA Kit (Ambion, Austin, TX) according to manufacturer's instruction. cDNA from the amplified RNA was synthesized as described previously.

Statistical analysis. All behavioral data were analyzed with a repeated measures ANOVA with Newman-Keuls test used for post hoc comparisons of individual means. A Student's $t$ test was used to compare ICER RT-PCR and real-time quantitative PCR data results between saline and DMI-treated wild-type mice. For CRF real-time quantitative PCR data, average differences in cycle thresholds were analyzed using a two-factor ANOVA, with Fisher's post hoc test used for comparisons of individual means. Plasma corticosterone data were also analyzed using a two-factor ANOVA and Fisher's post hoc test for comparison of individual means. Comparisons were considered statistically significant when $p$ values were $<0.05$.

\section{Results}

\section{DMI administration increases ICER expression}

Mice were treated subchronically or chronically with DMI to determine the effect of duration of antidepressant treatment on ICER expression in various regions of the brain. After subchronic DMI treatment, ICER expression was significantly upregulated in hypothalamic, but not frontal cortical or hippocampal regions of wild-type mice (Fig. 1) as determined by real-time quantitative PCR. Amplification plots demonstrate that the cycle threshold for ICER mRNA amplification in the hypothalamus is lower in DMI-treated wild-type mice, indicating that hypothalamic ICER expression is significantly upregulated after subchronic DMI administration compared with saline controls (Fig. 1a). When nor- 

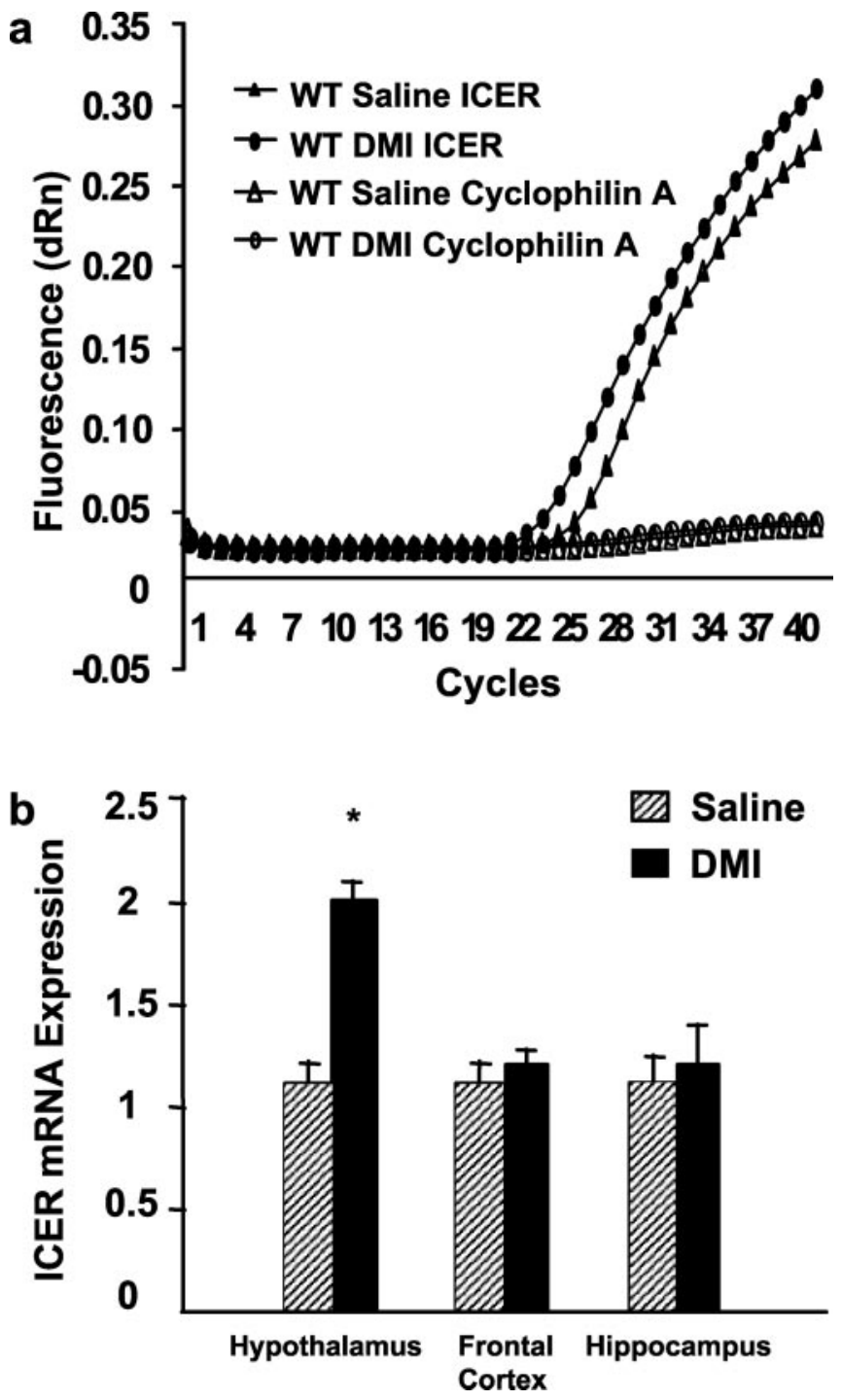

Figure 1. Real-time quantitative PCR analysis of ICER gene expression after subchronic antidepressant treatment. $a$, Steady-state levels of hypothalamic ICER and cyclophilin A (as internal control) mRNAs were determined by real-time quantitative PCR after three injections of saline or DMI in wild-type mice. Ten micrograms of total RNAs were reverse-transcribed into CDNA and amplified using primer pairs and fluorogenic probes specific to ICER and cyclophilin A, as described in Materials and Methods. Representative amplification plots of hypothalamic cDNA expression are shown for saline- and DMI-treated wild-type mice. Data is presented as fluorescence versus cycle number. Cycle threshold value is inversely proportional to the levels of endogenous gene expression. DMI administration significantly increased hypothalamic, but not frontal cortical or hippocampal ICER mRNA when compared with saline control (represented by an decreased cycle threshold number). No significant differences in cycle thresholds for cyclophilin A mRNA were observed between groups. Cycle thresholds for both ICER and cyclophilin A signals were 23-25 cycles, indicating similar levels of expression for these mRNAs within the tissues examined. $b$, Cycle thresholds normalized to cyclophilin A determined in $a$ were used to calculate ICER mRNA levels in hypothalamus, frontal cortex, and hippocampus and plotted relative to saline-treated wild-type mice (equal to 1). Subchronic DMI administration increased ICER mRNA levels by approximately twofold in wild-type mice compared with saline controls in the hypothalamus, but not in the frontal cortex or hippocampus. Student's $t$ test revealed the following difference: ${ }^{*} p<0.05$ compared with corresponding saline control.

malized to cyclophilin A expression, DMI-treated wild-type mice demonstrate an approximate twofold increase in ICER mRNA compared with saline controls (Fig. 1b). To examine the effects of chronic antidepressant administration on ICER expression, hypothalamic, frontal cortical, and hippocampal tissue from wildtype animals were evaluated after $21 \mathrm{~d}$ of DMI or saline treatment a

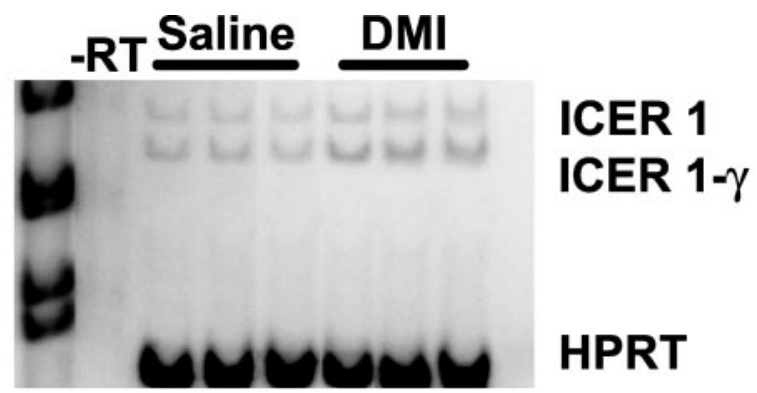

b

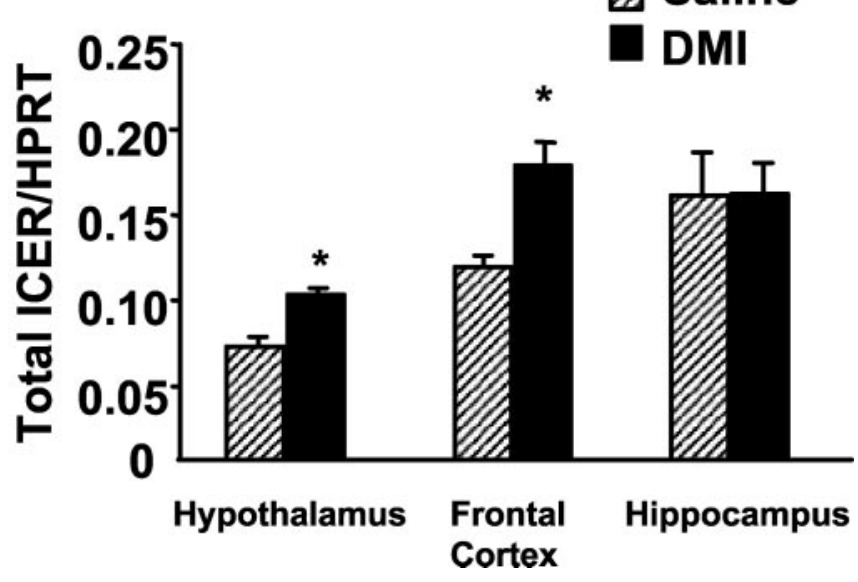

Figure 2. Reverse transcriptase PCR analysis of ICER gene expression after chronic antidepressant treatment. a, Steady-state levels of ICER and HPRT (as internal control) mRNAs in the hypothalamus of wild-type mice were determined by RT-PCR after $21 \mathrm{~d}$ of saline or DMI administration. Ten micrograms of total RNAs were reverse transcribed into CDNA and amplified using primer pairs specific to ICER and HPRT, as described in Materials and Methods. Three representative lanes are shown for saline and DMI treatment groups. ICER primers were designed to amplify both ICER-1 and splice variant ICER-1 $\gamma$, -RT, RNA-treated without reverse transcriptase enzyme as control. $b$, Signals for total ICER mRNA (ICER-1 + ICER-1 $\gamma$ ) were quantified using a Phosphorlmager and normalized to that of HPRT and plotted. DMI administration significantly increased ICER mRNA in the hypothalamus, frontal cortex, but not hippocampus of wild-type mice compared with saline controls. Student's $t$ test revealed the following difference: ${ }^{*} p<$ 0.05 compared with saline control.

using RT-PCR. After chronic administration of DMI, wild-type mice continued to exhibit significantly increased levels of ICER mRNA in the hypothalamus (Fig. 2). In contrast to subchronic DMI treatment, chronic DMI administration significantly elevated ICER mRNA levels in the frontal cortex (Fig. 2b). However, chronic DMI administration did not alter ICER expression in the hippocampus (Fig. 2b).

DMI administration reduces immobility in the FST and TST in wild-type and ICER-deficient mice

To test the role of ICER in DMI-mediated behavioral alterations in the FST, we administered saline and DMI (three doses over 24 hr: 10,10 , and $20 \mathrm{mg} / \mathrm{kg}$, s.c.) to wild-type and ICER-deficient mice and measured immobility. Subchronic administration of DMI reduced immobility times in wild-type and mutant mice to similar levels compared with respective saline controls (Fig. 3). There is no quantifiable difference in basal locomotion of mutant mice compared with wild-type mice when tested in home cage activity monitors (data not shown). To examine the effects of chronic DMI treatment on FST immobility, animals were maintained on twice daily injections of DMI or saline $(10 \mathrm{mg} / \mathrm{kg}$, i.p.) for $21 \mathrm{~d}$ and re-evaluated in the FST on day 21 (Fig. 3). With 


\section{Saline - DMI}

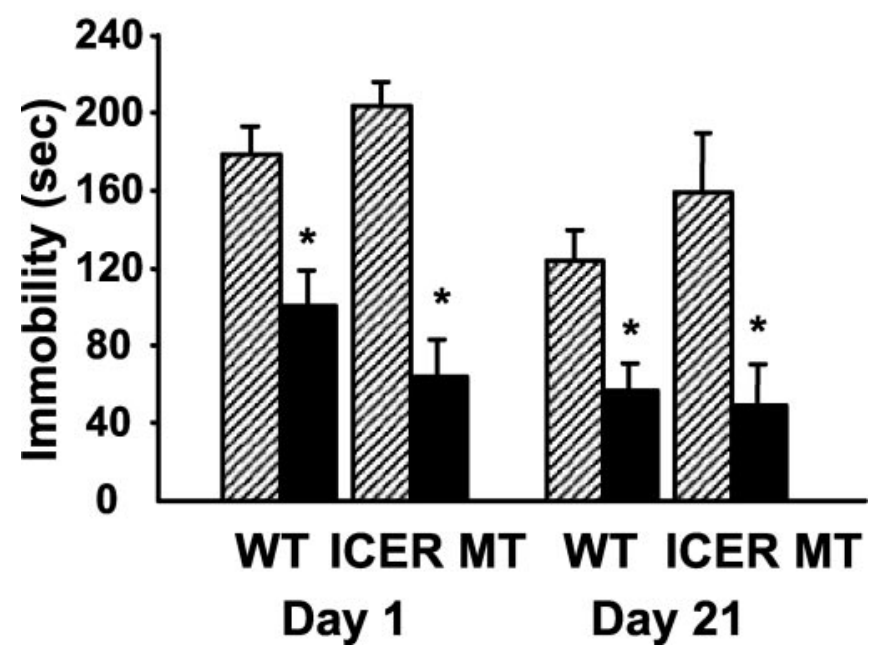

Figure 3. Behavioral response of wild-type and ICER-deficient mice in the forced swim test after antidepressant treatment. Animals were administered saline or DMI twice daily $(10 \mathrm{mg} /$ $\mathrm{kg}$, i.p.) with $20 \mathrm{mg} / \mathrm{kg}$ subcutaneously administered on testing days and evaluated for immobility in the FST. DMI treatment significantly reduced immobility times in both wild-type and ICER-deficient mice on day 1 (subchronic) and 21 (chronic) of the treatment paradigm compared with respective saline controls. Results are presented as mean immobility \pm SEM (in seconds). Repeated measures ANOVA and post hoc Newman-Keuls pairwise comparisons revealed the following differences: ${ }^{*} p<0.05$ compared with corresponding saline control.

chronic DMI administration, both wild-type and ICER-deficient mice maintained significantly reduced immobility times over the course of the study compared with respective saline controls.

Although the FST is generally an effective screen for measuring the behavioral effects antidepressant drugs, it is not reliable for testing selective serotonin reuptake inhibitors in mice with this genetic background (Lucki, 2001). Therefore, mice were examined using the selective serotonin reuptake inhibitor fluoxetine in the tail suspension test to rule out effects specific to tricyclic antidepressants. Responses were compared with those of DMI in the same testing session. Similar to the FST, acute and chronic antidepressant treatment reduced immobility times in wild-type and ICER-deficient mice in the TST compared with respective saline controls (Fig. 4). Together these data demonstrate that behavioral effects do not habituate throughout the course of a chronic drug treatment in ICER-deficient mice and that ICER is not required for the manifestation of behavioral responses after acute or chronic DMI or fluoxetine administration.

\section{ICER is required for DMI-mediated suppression of} corticosterone release after FST

Unlike the TST, the FST is a potent activator of the HPA axis (Connor et al., 2000). Therefore, to examine the stress-induced endocrine response in ICER-deficient animals, blood samples were taken $20 \mathrm{~min}$ and $1 \mathrm{hr}$ after FST exposure for plasma corticosterone analysis after subchronic (Fig. 5a) and chronic (Fig. 5b) DMI treatment. Corticosterone was significantly elevated in both wild-type and ICER-deficient animals at $20 \mathrm{~min}$ after forced swimming compared with non-swim stress controls (Fig. 5). Corticosterone concentrations returned to control levels by $1 \mathrm{hr}$ after swim stress in both groups (data not shown). These data demonstrate that ICER is not essential for the stress-induced en-

\section{valine $\square$ FLX}

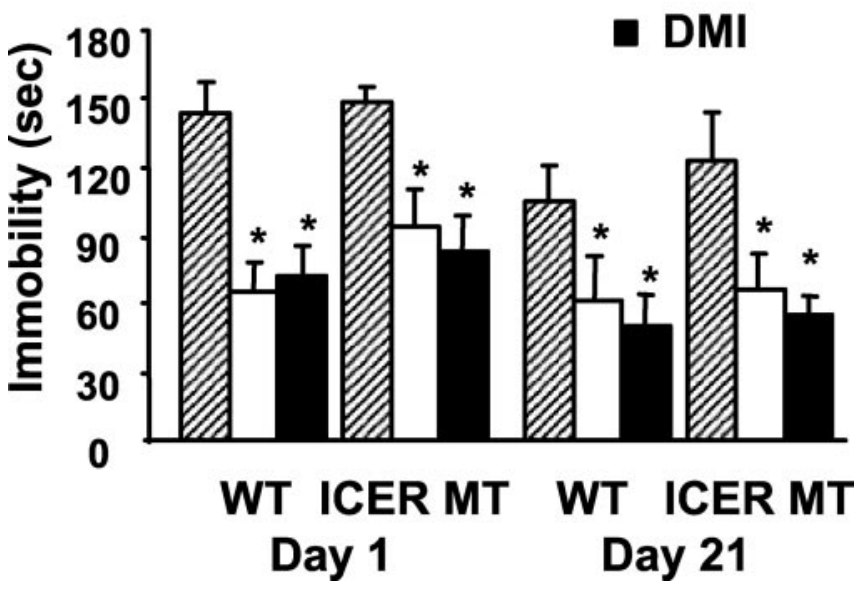

Figure 4. Behavioral response of wild-type and ICER-deficient mice in the tail suspension test after antidepressant treatment. Animals were administered saline, DMl, or FLX ( $20 \mathrm{mg} / \mathrm{kg}$, i.p.) 30 min before a 6 min test session and evaluated for immobility in the TST. Between testing days, animals were maintained on twice daily saline or DMI (10 mg/kg, i.p.) injections. Acute (day 1) and chronic (day 21) FLX and DMI significantly reduced immobility times in both wildtype and ICER-deficient mice compared with respective saline controls. Results are presented as mean immobility \pm SEM (sec). ANOVA and post hoc Newman-Keuls pairwise comparisons revealed the following differences: ${ }^{*} p<0.05$ compared with corresponding saline control.

docrine response after swim exposure. Both subchronic (Fig. $5 a$ ) and chronic (Fig. 5b) DMI administration significantly attenuated swim stress-induced elevations in plasma corticosterone in wild-type mice compared with saline-treated stress controls. However, neither subchronic nor chronic DMI treatment was able to suppress increased corticosterone concentrations in ICER-deficient mice after FST exposure (Fig. 5).

\section{ICER is required for DMI mediated downregulation of CRF} mRNA in the hypothalamus

CRF regulates corticosterone release and may also mediate the effects of DMI on HPA axis activity. Therefore, to elucidate the molecular mechanism by which DMI suppresses corticosterone release acutely after swim stress, mice were examined for alterations in hypothalamic CRF expression using real-time quantitative PCR. Amplification plots demonstrate that the cycle threshold for CRF mRNA amplification is greater in DMI-treated wildtype mice, indicating that CRF expression is significantly downregulated in wild-type mice treated chronically with DMI after FST exposure compared with saline controls (Fig. 6a). When normalized to TBP expression, DMI-treated wild-type mice demonstrate an approximate twofold reduction in CRF mRNA compared with wild-type saline controls (Fig. 6b). However, this DMI-induced downregulation of CRF expression did not occur in ICER mutant mice. CRF mRNA levels in DMItreated ICER-deficient mice were equivalent to saline-treated wild-type and mutant mice exposed to swim stress (Fig. 6b). These data suggest that ICER is critical to the mechanism by which DMI reduces CRF mRNA. To verify the presence of ICER within the region of CRF-expressing neurons in the PVN (Fig. $7 a$ ), these neurons were microdissected from wild-type mice (Fig. $7 b$ ) and examined for ICER expression using real-time quantitative PCR (Fig. 7c). The results provide evidence for concurrent expression of CRF, ICER, and cyclophilin A in cells of the PVN. Together, these data indicate that ICER, acting as a potent CRF 

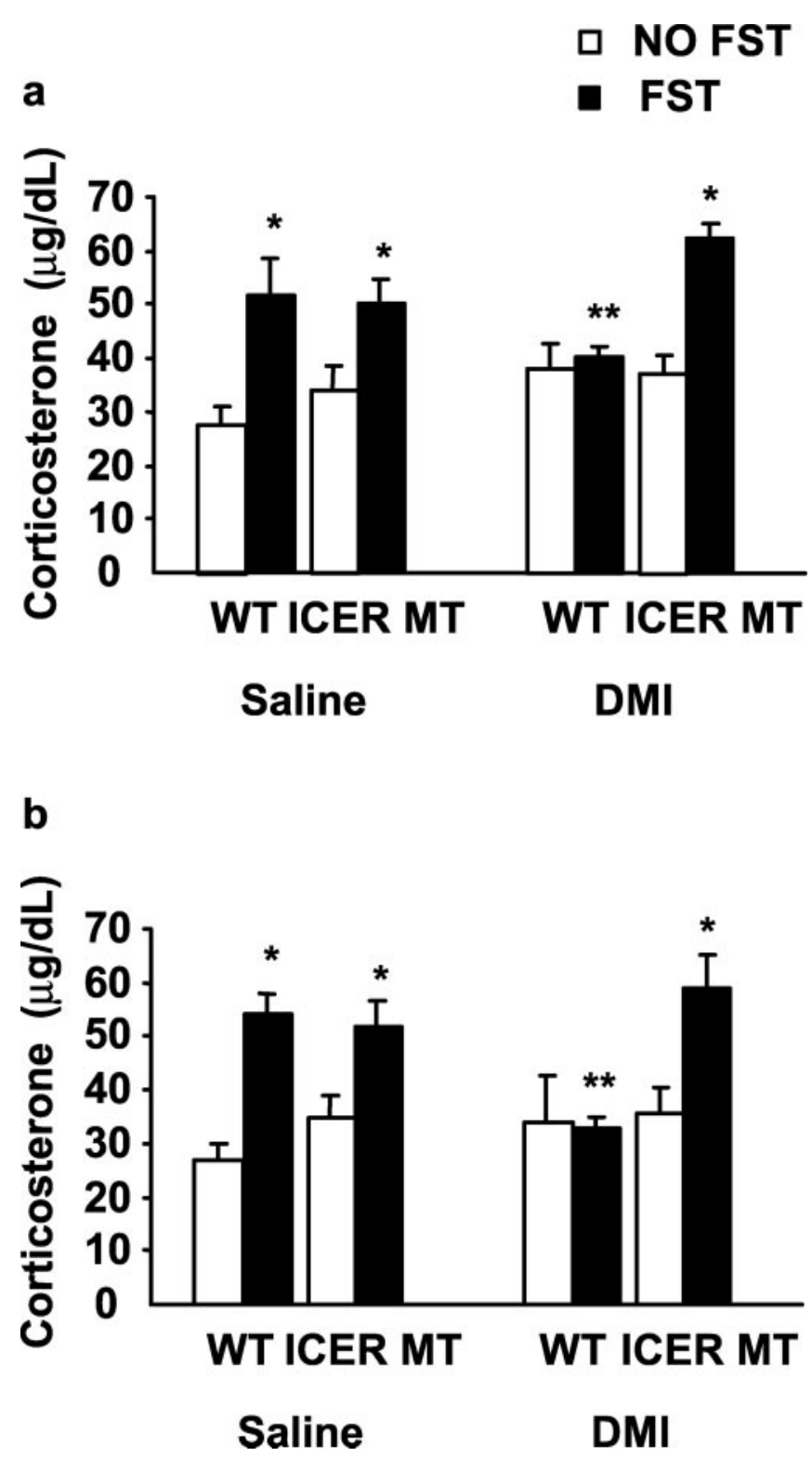

Figure 5. Plasma corticosterone concentration after exposure to the forced swim test. Animals were examined for changes in the stress hormone corticosterone with and without FST exposure. In wild-type and ICER mutant mice, swim stress induced a significant elevation in corticosterone levels compared with respective non-swim stressed control mice 20 min after swim exposure on day $1(a)$ and day $21(b)$. Administration of subchronic $(a)$ and chronic $(b)$ DMI significantly blunted the elevation in corticosterone in wild-type mice compared with saline-treated swim-stressed controls. In ICER mutant mice, however, neither subchronic ( $a$ ) nor chronic (b) DMl administration was able to suppress swim stress-induced elevations in corticosterone. Values are presented as mean corticosterone \pm SEM (in micrograms per decaliter). Results are presented as mean immobility \pm SEM (in seconds). ANOVA and Fisher's post hoc test for comparison of individual means revealed the following differences: ${ }^{*} p<0.05$ compared with corresponding non-swim stress control and ${ }^{* *} p<0.05$ compared with corresponding swim stress saline control.

repressor, is critical to the mechanism by which DMI regulates corticosterone secretion after swim stress.

\section{Discussion}

The cAMP-protein kinase A pathway has been identified as an important signaling system involved in the effects of stress and repeated antidepressant drug administration. Within this pathway the transcription factor CREB has been well studied (Nibuya

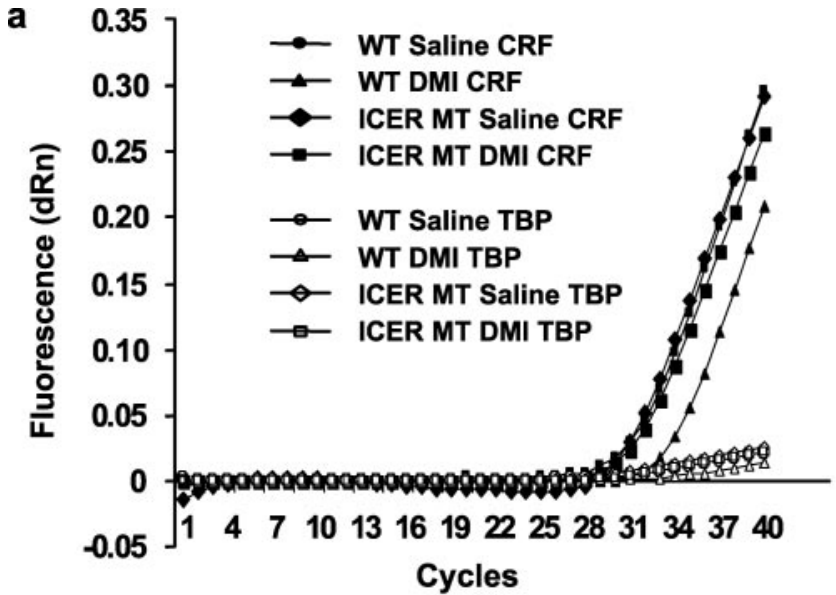

b

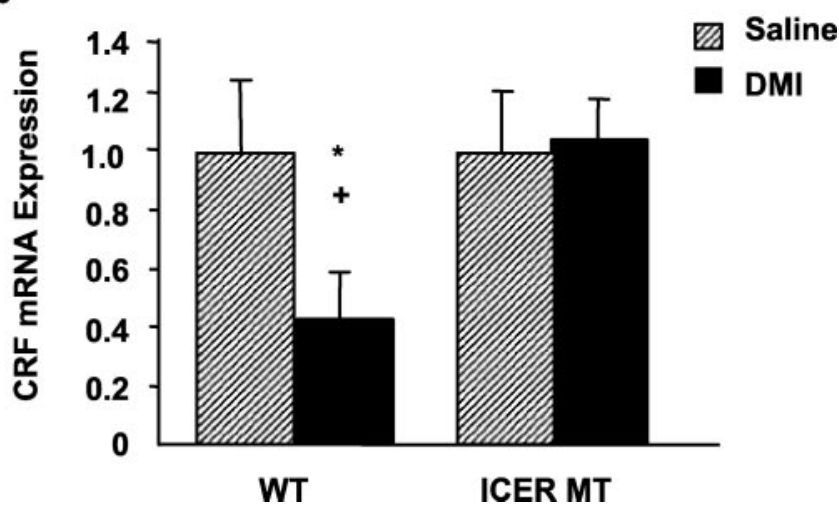

Figure 6. Real-time quantitative $P C R$ analysis of CRF gene expression after exposure to swim stress. a, Steady-state levels of hypothalamic CRF and TBP (as internal control) mRNAs were determined by real-time quantitative PCR after $21 \mathrm{~d}$ of saline or DMI administration in wild-type or ICER-deficient mice. Ten micrograms of total RNAs were reverse-transcribed into CDNA and amplified using primer pairs and fluorogenic probes specific to CRF and TBP as described in Materials and Methods. Representative amplification plots are shown for saline and DMI treatment groups for wild-type and ICER-deficient mice. Data is presented as fluorescence versus cycle number. Cycle threshold value is inversely proportional to the levels of endogenous gene expression. DMI administration significantly reduced CRF mRNA after swim stress compared with wild-type saline control (represented by an increased cycle threshold number). DMI was unable to blunt CRF mRNA expression in ICER-deficient mice compared with saline controls. № significant differences in TBP mRNA were observed between groups. Cycle thresholds for both CRF and TBP signals were 23-25 cycles, indicating similar levels of expression for these mRNAs within the tissues examined. $b$, Cycle thresholds normalized to TBP determined in $a$ were used to calculate the CRF mRNA levels and plotted relative to saline-treated wild-type mice (equal to 1). DMI reduced CRF mRNA levels by approximately twofold in wild-type mice compared with wild-type saline controls. ANOVA and post hoc Fisher's pairwise comparisons revealed the following differences: ${ }^{*} p<0.05$ compared with corresponding saline control and $+p<0.05$ compared with DMI-treated ICER-deficient mice.

et al., 1996; Conti et al., 2002), however the impact of a highly related protein, CREM, is not well described. The CREM gene encodes a variety of isoforms through alternative splicing that function as either activators or repressors of cAMP-responsive transcription (Foulkes et al., 1991, 1992; Delmas et al., 1992; Foulkes and Sassone-Corsi, 1992; Laoide et al., 1993). Deletion of the CREM gene in the mouse results in the loss of all of these isoforms and causes male mice to be sterile (Blendy et al., 1996; Nantel et al., 1996). This phenotype most likely results from deletion of the CREM activator isoform CREM $\tau$, which is highly expressed in testes (Foulkes et al., 1992). The functional consequences resulting from deletion of repressor isoforms, such as ICER, however, have not been examined in these mice. In contrast to CREM $\tau$ activity, ICER is suggested to regulate compo- 
a

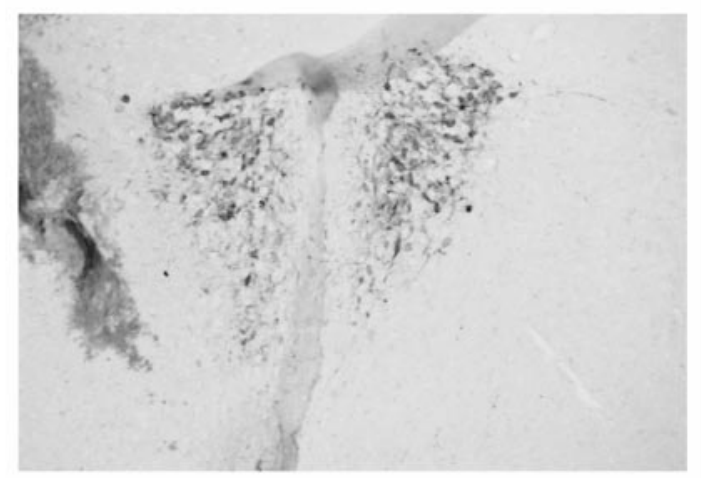

\section{b}

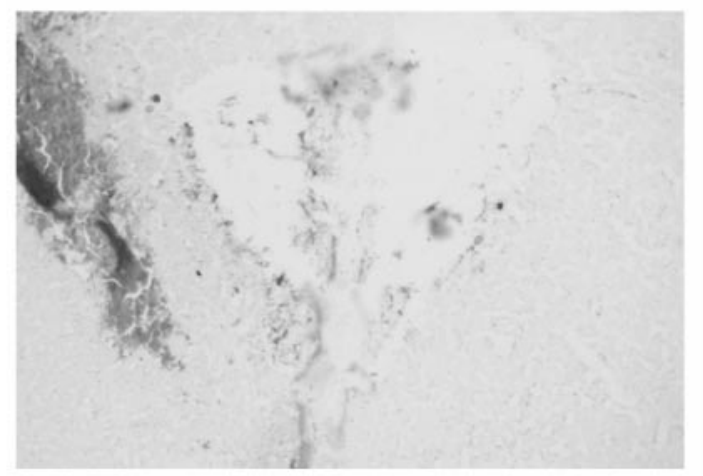

c

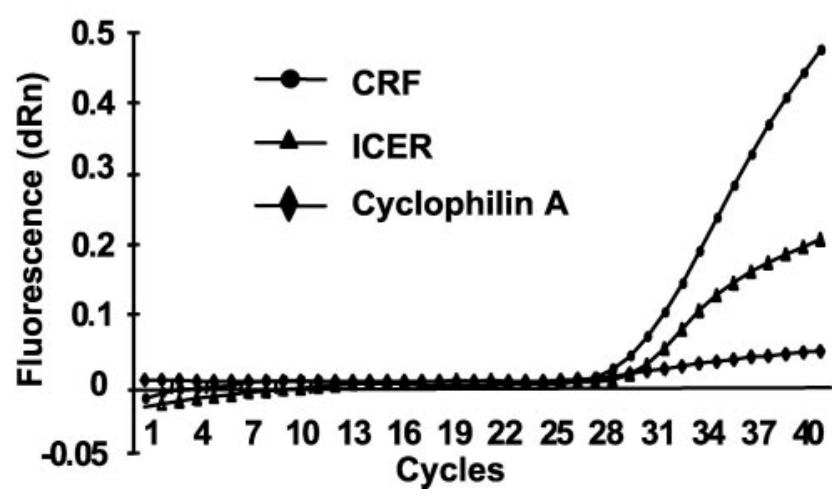

Figure 7. Colocalization of ICER and CRF mRNA in PVN neurons of the hypothalamus. $a$, CRF-expressing PVN neurons in wild-type mice were identified using immunohistochemical techniques as described in Materials and Methods. b, CRF-expressing cells in the PVN were microdissected and processed for aRNA amplification and CDNA synthesis. C, CRF-expressing cells were examined for ICER coexpression using real-time quantitative PCR. aRNA from CRFpositive PVN cells was reverse transcribed into CDNA and amplified using primer pairs and fluorogenic probes specific to CRF, ICER, and cyclophilin A (as internal control), as described in Materials and Methods. Representative amplification plots are shown. Data is presented as fluorescence versus cycle number.

nents of the HPA axis and limbic system (Fitzgerald et al., 1996a; Della Fazia et al., 1998; Mazzucchelli and Sassone-Corsi, 1999). The ICER protein consists primarily of a basic-leucine zipper region, which allows heterodimerization with all CREM and CREB proteins, resulting in nonactivating dimers (Stehle et al., 1993). The presence of four tandem CRE sequences in the ICER promoter suggests cAMP inducibility, therefore ICER is classified as an early response gene (Molina et al., 1993).

Previous studies have suggested a role for regulation of early response genes, like c-fos, c-jun, and ICER, by antidepressant treatments. Acute DMI administration (Dahmen et al., 1997) as well as electroconvulsive seizure (ECS) (Cole et al., 1990) have been shown to induce immediate early gene expression in the rat brain, however, to date only ECS has been shown to significantly upregulate ICER expression in the rat frontal cortex and hippocampus. ICER is also sensitive to adrenergic stimulation, which is a characteristic of most tricyclic antidepressants. In line with this evidence, we demonstrate here that ICER expression is significantly increased after chronic DMI treatment in mouse frontal cortex, but not hippocampus. Furthermore, after both subchronic and chronic DMI treatment, ICER expression is increased in the hypothalamus. Preliminary studies indicate that chronic fluoxetine $(10 \mathrm{mg} / \mathrm{kg})$ does not alter levels of ICER in either frontal cortex or hippocampus (data not shown), however regulation in the hypothalamus has not been examined. These data indicate that ICER may be a critical molecular mediator of tricyclic antidepressant function.

One approach to identify genes that may contribute to a psychiatric illness, such as depression, involves the genetic dissection of complex phenotypes into endophenotypes, measurable biological traits that are associated with target disease phenotypes. Clinically, depression involves alterations in behavioral as well as endocrine responses, however the functional relationship that exists between these two responses is not well defined. Therefore, in our animal model, we examined both the behavioral responses associated with antidepressant administration as well as the endocrine alterations associated with desipramine treatment to evaluate if ICER is a molecular mediator of one or both of these outcome measures.

Behavioral models, such as the FST and TST, are widely used for preclinical assessment of antidepressant activity (Cryan et al., 2002). We evaluated ICER-deficient mice in both paradigms to determine if ICER is involved in mediating DMI-induced behaviors. ICER-deficient mice respond to DMI similarly to wild-type mice in the FST and TST, indicating that ICER is not required for the expression of the behavioral responses to antidepressant drug treatment. These data parallel previous studies from our laboratory demonstrating that CREB deficiency does not compromise behavioral responses to antidepressant drugs (Conti et al., 2002). However, in contrast to CREB-deficient mice, which demonstrate a baseline change in immobility behavior, ICER-deficient mice have similar levels of immobility as wild-type controls in both the FST and TST. Furthermore, similar to wild-type controls, ICER-deficient mice exhibited a significant reduction in immobility after both DMI and FLX in the tail suspension test, indicating a general responsivity of ICER-deficient mice to at least two classes of antidepressant drugs.

Psychiatric morbidity associated with depression can often be accompanied or even precipitated by stress. There is close clinical and biochemical resemblance between depressive symptoms and the response to stressful experiences, which led to the hypothesis that depression represents activation of the primary mediators of the stress response. As a result, dysregulation of the HPA axis has been implicated in depression and its treatment. Clinical data indicates that a subset of patients with depression exhibit hyperactivity of the HPA axis, which is normalized after successful antidepressant therapy (Asnis et al., 1992; Holsboer-Trachsler et al., 1994; Gold et al., 1995; Heuser et al., 1996; Arborelius et al., 1999; Sapolsky, 2000).

Previous reports suggest that ICER induction is coupled to the HPA axis in response to stress (Della Fazia et al., 1998; Mazzucchelli and Sassone-Corsi, 1999). Stress associated with the FST activates the HPA axis and causes significant elevations of corticosterone (Connor et al., 2000). However, in the present study we demonstrate that both wild-type and ICER-deficient mice exhibit 
similar elevations in serum corticosterone concentration after swim stress compared with respective non-stress controls, indicating that ICER is not critical for the corticosterone response to swim stress. This finding parallels previous studies that report normal responses of CREM mutant mice to behavioral stress (Maldonado et al., 1999).

Furthermore, our data demonstrate that swim stress-induced elevation in corticosterone concentration is attenuated by DMI administration in wild-type mice. These data are in agreement with previous studies in the rat that document the attenuation of swim stress associated elevation in serum corticosterone by DMI (Connor et al., 2000; Conti et al., 2002), but not by selective serotonin reuptake inhibitors (Connor et al., 2000). In contrast, swim stress-induced elevation in corticosterone is not blocked by DMI administration in ICER-deficient mice, indicating that ICER is critical to the mechanism by which DMI mediates HPA axis activity. In addition, these findings also indicate that the molecular mechanisms that activate stress responses after the FST, which are unaffected in ICER-deficient mice, are distinct from those used by DMI to suppress these responses.

DMI treatment is suggested to reduce serum corticosterone concentrations by a variety of mechanisms, including suppression of CRF expression (Brady et al., 1991; Centeno and Volosin, 1997). Using real-time quantitative PCR we demonstrate that chronic DMI treatment significantly decreases CRF mRNA expression in the hypothalamus of wild-type mice after swim stress. Chronic DMI treatment also significantly increases hypothalamic ICER mRNA expression. In contrast, ICER-deficient mice fail to demonstrate reductions in swim stress-induced CRF expression after DMI treatment. Together these data suggest that ICER negatively regulates hypothalamic CRF mRNA expression. In further support of these findings, we demonstrate that ICER is enriched in CRF-expressing cells of the PVN, suggesting the potential for a direct interaction between this transcription factor and its target gene. Indeed, the presence of a CRE element in the promoter region of the CRF gene indicates that regulation may occur via a CRE-binding mechanism (Meyer et al., 1993). Recent studies in two hypothalamic cell lines demonstrate that cotransfection of ICER with a luciferase reporter gene driven by a CRF promoter fragment markedly inhibited basal and forskolinstimulated CRF promoter activity (Kalitchenko et al., 2003; Liu et al., 2003). Together, these data reveal a novel role for ICER as a strong transcriptional repressor of CRF, and that ICER expression may result in downregulation of CRF mRNA and subsequently, attenuated corticosterone secretion.

Response to antidepressant treatment can be observed at the behavioral and endocrine levels. The relationship between the underlying mechanisms associated with these responses and the impact they have on clinical outcome is complex and may contribute to the variability in efficacy often observed with antidepressant treatment. We have used a combination of approaches to examine the role of CRE transcription factors in mediating the behavioral and endocrine consequences of antidepressant treatment. Previously, we have shown that an activator of CREmediated gene expression, CREB, is not required for behavioral responses to antidepressant drugs (Conti et al., 2002). In a similar manner, we now demonstrate that a strong repressor of CREmediated gene expression, ICER, is dispensable for behavioral responses. In contrast, although CREB deficiency does not affect the endocrine response to antidepressant treatment, an ICER deficiency clearly compromises this response. Hence, antidepressant modulation of the HPA axis may depend more on repression (ICER) of CRE-mediated gene expression than on activation
(CREB). Whether or not HPA axis abnormalities are a primary cause of depression, stress is clearly an important causative factor in the development or expression of depressive symptoms. For this reason, new developments in antidepressant medications have focused on CRF receptor antagonists (Arborelius et al., 1999; O'Brien et al., 2001). In a similar manner, identification of the molecules associated with downregulation of the CRF gene, such as ICER, may identify new targets for novel antidepressant drugs.

\section{References}

Arborelius L, Owens MJ, Plotsky PM, Nemeroff CB (1999) The role of corticotropin-releasing factor in depression and anxiety disorders. J Endocrinol 160:1-12.

Asnis GM, Sanderson WC, van Praag HM (1992) Cortisol response to intramuscular desipramine in patients with major depression and normal control subjects: a replication study. Psychiatry Res 44:237-250.

Blendy JA, Kaestner KH, Weinbauer GF, Nieschlag E, Schutz G (1996) Severe impairment of spermatogenesis in mice lacking the CREM gene. Nature 380:162-165.

Brady LS, Whitfield Jr HJ, Fox RJ, Gold PW, Herkenham M (1991) Longterm antidepressant administration alters corticotropin-releasing hormone, tyrosine hydroxylase, and mineralocorticoid receptor gene expression in rat brain. Therapeutic implications. J Clin Invest 87:831-837.

Centeno VA, Volosin M (1997) Chronic treatment with desipramine: effect on endocrine and behavioral responses induced by inescapable stress. Physiol Behav 62:939-944.

Cole AJ, Abu-Shakra S, Saffen DW, Baraban JM, Worley PF (1990) Rapid rise in transcription factor mRNAs in rat brain after electroshock-induced seizures. J Neurochem 55:1920-1927.

Connor TJ, Kelliher P, Shen Y, Harkin A, Kelly JP, Leonard BE (2000) Effect of subchronic antidepressant treatments on behavioral, neurochemical, and endocrine changes in the forced-swim test. Pharmacol Biochem Behav 65:591-597.

Conti AC, Cryan JF, Dalvi A, Lucki I, Blendy JA (2002) cAMP response element-binding protein is essential for the upregulation of brain-derived neurotrophic factor transcription, but not the behavioral or endocrine responses to antidepressant drugs. J Neurosci 22:3262-3268.

Cryan JF, Markou A, Lucki I (2002) Assessing antidepressant activity in rodents: recent developments and future needs. Trends Pharmacol Sci 23:238-245.

Dahmen N, Fehr C, Reuss S, Hiemke C (1997) Stimulation of immediate early gene expression by desipramine in rat brain. Biol Psychiatry 42:317-323.

Della Fazia MA, Servillo G, Foulkes NS, Sassone-Corsi P (1998) Stressinduced expression of transcriptional repressor ICER in the adrenal gland. FEBS Lett 434:33-36.

Delmas V, Laoide BM, Masquilier D, de Groot RP, Foulkes NS, Sassone-Corsi $P$ (1992) Alternative usage of initiation codons in mRNA encoding the cAMP-responsive-element modulator generates regulators with opposite functions. Proc Natl Acad Sci USA 89:4226-4230.

Dowlatshahi D, MacQueen GM, Wang JF, Young LT (1998) Increased temporal cortex CREB concentrations and antidepressant treatment in major depression. Lancet 352:1754-1755.

Dowlatshahi D, MacQueen GM, Wang JF, Reiach JS, Young LT (1999) G Protein-coupled cyclic AMP signaling in postmortem brain of subjects with mood disorders: effects of diagnosis, suicide, and treatment at the time of death. J Neurochem 73:1121-1126.

Fitzgerald LR, Vaidya VA, Terwilliger RZ, Duman RS (1996a) Electroconvulsive seizure increases the expression of CREM (cyclic AMP response element modulator) and ICER (inducible cyclic AMP early repressor) in rat brain. J Neurochem 66:429-432.

Fitzgerald LR, Li Z, Machida CA, Fishman PH, Duman RS (1996b) Adrenergic regulation of ICER (inducible cyclic AMP early repressor) and beta1-adrenergic receptor gene expression in C6 glioma cells. J Neurochem 67:490-497.

Foulkes NS, Sassone-Corsi P (1992) More is better: activators and repressors from the same gene. Cell 68:411-414.

Foulkes NS, Borrelli E, Sassone-Corsi P (1991) CREM gene: use of alternative DNA-binding domains generates multiple antagonists of cAMPinduced transcription. Cell 64:739-749. 
Foulkes NS, Mellstrom B, Benusiglio E, Sassone-Corsi P (1992) Developmental switch of CREM function during spermatogenesis: from antagonist to activator. Nature 355:80-84.

Gold PW, Licinio J, Wong ML, Chrousos GP (1995) Corticotropin releasing hormone in the pathophysiology of melancholic and atypical depression and in the mechanism of action of antidepressant drugs. Ann NY Acad Sci 771:716-729.

Gonzalez GA, Montminy MR (1989) Cyclic AMP stimulates somatostatin gene transcription by phosphorylation of CREB at serine 133. Cell 59:675-680.

Heuser IJ, Schweiger U, Gotthardt U, Schmider J, Lammers CH, Dettling M, Yassouridis A, Holsboer F (1996) Pituitary-adrenal-system regulation and psychopathology during amitriptyline treatment in elderly depressed patients and normal comparison subjects. Am J Psychiatry 153:93-99.

Holsboer-Trachsler E, Hemmeter U, Hatzinger M, Seifritz E, Gerhard U, Hobi V (1994) Sleep deprivation and bright light as potential augmenters of antidepressant drug treatment-neurobiological and psychometric assessment of course. J Psychiatry Res 28:381-399.

Kalitchenko N, Liu Y, Kasckow J, Aguilera G (2003) Self-limitation of cAMP-stimulated CRH transcription in the hypothalamic cell line 4B: role of inducible cAMP early repressor (ICER). Soc Neurosci Abstr 29:15.11.

Lalli E, Sassone-Corsi P (1994) Signal transduction and gene regulation: the nuclear response to cAMP. J Biol Chem 269:17359-17362.

Laoide BM, Foulkes NS, Schlotter F, Sassone-Corsi P (1993) The functional versatility of CREM is determined by its modular structure. EMBO J 12:1179-1191.

Lee KA, Masson N (1993) Transcriptional regulation by CREB and its relatives. Biochim Biophys Acta 1174:221-233.

Liu Y, Shepard JD, Morales M, Sassone-Corsi P, Aguilera G (2003) Involvement of glucocorticoids and inducible isoforms of CREM in the termination of corticotropin releasing hormone transcriptional responses to stress. Soc Neurosci Abstr 29:15.10.

Livak KJ, Schmittgen TD (2001) Analysis of relative gene expression data using real-time quantitative PCR and the 2(-Delta Delta C(T)) Method. Methods 25:402-408.

Lucki I (2001) A prescription to resist proscriptions for murine models of depression. Psychopharmacology (Berl) 153:395-398.

Lucki I, Dalvi A, Mayorga AJ (2001) Sensitivity to the effects of pharmacologically selective antidepressants in different strains of mice. Psychopharmacology (Berl) 155:315-322.

Luckman SM, Cox HJ (1995) Expression of inducible cAMP early repressor (ICER) in hypothalamic magnocellular neurons. Brain Res Mol Brain Res 34:231-238.

Maldonado R, Smadja C, Mazzucchelli C, Sassone-Corsi P, Mazucchelli C (1999) Altered emotional and locomotor responses in mice deficient in the transcription factor CREM. Proc Natl Acad Sci USA 96:14094-14099.

Mazzucchelli C, Sassone-Corsi P (1999) The inducible cyclic adenosine monophosphate early repressor (ICER) in the pituitary intermediate lobe: role in the stress response. Mol Cell Endocrinol 155:101-113.

Mellstrom B, Naranjo JR, Foulkes NS, Lafarga M, Sassone-Corsi P (1993) Transcriptional response to cAMP in brain: specific distribution and induction of CREM antagonists. Neuron 10:655-665.

Meyer TE, Waeber G, Lin J, Beckmann W, Habener JF (1993) The promoter of the gene encoding $3^{\prime}, 5^{\prime}$-cyclic adenosine monophosphate (cAMP) response element binding protein contains cAMP response elements: evidence for positive autoregulation of gene transcription. Endocrinology 132:770-780

Molina CA, Foulkes NS, Lalli E, Sassone-Corsi P (1993) Inducibility and negative autoregulation of CREM: an alternative promoter directs the expression of ICER, an early response repressor. Cell 75:875-886.

Nantel F, Monaco L, Foulkes NS, Masquilier D, LeMeur M, Henriksen K, Dierich A, Parvinen M, Sassone-Corsi P (1996) Spermiogenesis deficiency and germ-cell apoptosis in CREM-mutant mice. Nature 380:159-162.

Nibuya M, Nestler EJ, Duman RS (1996) Chronic antidepressant administration increases the expression of cAMP response element binding protein (CREB) in rat hippocampus. J Neurosci 16:2365-2372.

O'Brien D, Skelton KH, Owens MJ, Nemeroff CB (2001) Are CRF receptor antagonists potential antidepressants? Hum Psychopharmacol 16:81-87.

Porsolt RD, Le Pichon M, Jalfre M (1977) Depression: a new animal model sensitive to antidepressant treatments. Nature 266:730-732.

Sapolsky RM (2000) Glucocorticoids and hippocampal atrophy in neuropsychiatric disorders. Arch Gen Psychiatry 57:925-935.

Silva AJ, Simpson EM, Takahashi JS, Lipp H-P, Nakanishi S, Wehner JM, Giese KP, Tully T, Abel T, Chapman PF, Fox K, Grant S, Itohara S, Lathe R, Mayford M, McNamara JO, Morris RJ, Picciotto M, Roder J, Shin H-S, Slessinger PA, Storm DR, Stryker MP, Tonegawa S, Wang Y, Wolfer DP (1997) Mutant mice and neuroscience: Recommendations concerning genetic background. Neuron 19:755-759.

Stehle JH, Foulkes NS, Molina CA, Simonneaux V, Pevet P, Sassone-Corsi P (1993) Adrenergic signals direct rhythmic expression of transcriptional repressor CREM in the pineal gland. Nature 365:314-320.

Steru L, Chermat R, Thierry B, Simon P (1985) The tail suspension test: a new method for screening antidepressants in mice. Psychopharmacology 85:367-370.

Sunal R, Gumusel B, Kayaalp SO (1994) Effect of changes in swimming area on results of "behavioral despair test". Pharmacol Biochem Behav 49:891-896.

Tamura T, Sumita K, Fujino I, Aoyama A, Horikoshi M, Hoffmann A, Roeder RG, Muramatsu M, Mikoshiba K (1991) Striking homology of the "variable" N-terminal as well as the "conserved core" domains of the mouse and human TATA-factors (TFID). Nucleic Acids Res 19:3861-3865.

Thome J, Sakai N, Shin K, Steffen C, Zhang YJ, Impey S, Storm D, Duman RS (2000) cAMP response element-mediated gene transcription is upregulated by chronic antidepressant treatment. J Neurosci 20:4030-4036.

Valentino RJ, Page M, Van Bockstaele E, Aston-Jones G (1992) Corticotropinreleasing factor innervation of the locus coeruleus region: distribution of fibers and sources of input. Neuroscience 48:689-705.

Van Bockstaele EJ, Colago EE, Valentino RJ (1996) Corticotropin-releasing factor-containing axon terminals synapse onto catecholamine dendrites and may presynaptically modulate other afferents in the rostral pole of the nucleus locus coeruleus in the rat brain. J Comp Neurol 364:523-534.

Wilson PA, Melton DA (1994) Mesodermal patterning by an inducer gradient depends on secondary cell-cell communication. Curr Biol $4: 676-686$ 